\title{
Large-Scale Direct Patterning of Aligned Single-Walled Carbon Nanotube Arrays Using Dip-Pen Nanolithography
}

\author{
Jae-Hyeok Lee, ${ }^{* \dagger}$ Choolakadavil Khalid Najeeb, ${ }^{\ddagger}$ Gwang-Hyeon Nam, ${ }^{\ddagger}$ Yonghun Shin, ${ }^{\S}$ Jung- \\ Hyurk Lim," and Jae-Ho Kim ${ }^{* \neq}$ \\ †Department of Materials Science and Engineering, Northwestern University, 2220 Campus Drive, Evanston, Illinois 60208, \\ United States \\ \$Department of Molecular Science and Technology, Ajou University, Suwon, 443-749, Republic of Korea

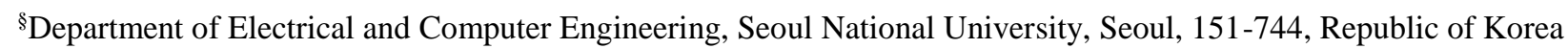 \\ "Department of Polymer Science and Technology, Korea National University of Transportation, Chungju 380-702, Republic \\ of Korea \\ *E-mail: jae-hyeok.lee@northwestern.edu, jhkim@ajou.ac.kr
}

\section{Table of Contents}

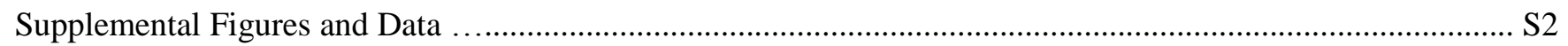

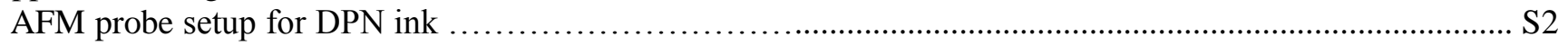

Stepwise evaluation of SWNTs based electrodes fabrication .................................................................. S3

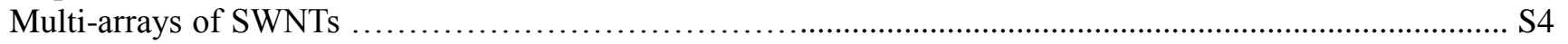




\section{Supplemental Figures and Data}

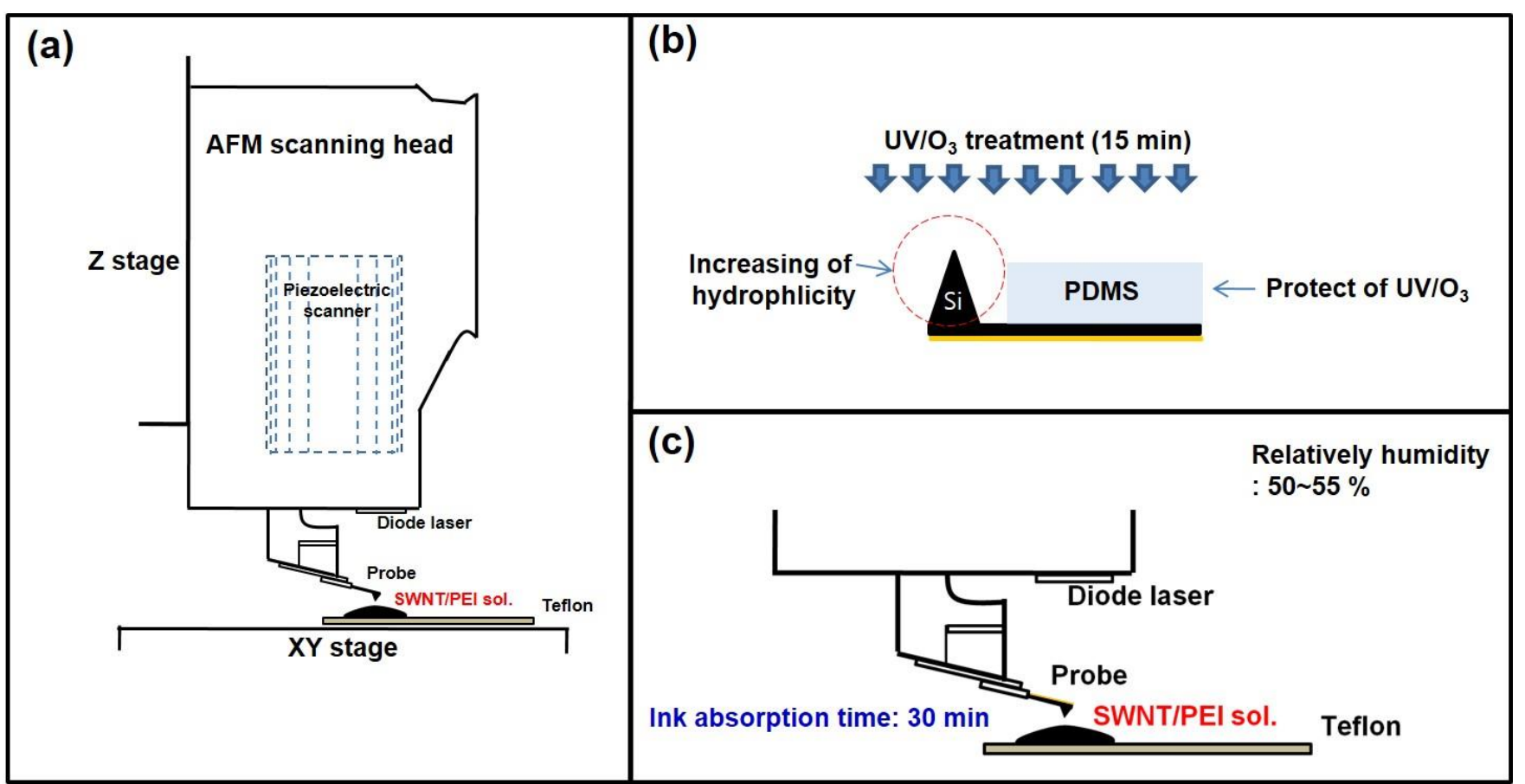

Figure S1. AFM probe setup for DPN inking. (a) A schematic illustration of the loading of SWNT/DPN ink onto the AFM probe. (b) UV-ozone treatment was applied to the AFM probe to increase the loading amount of SWNT/DPN ink. The AFM probe body is surrounded by polydimethylsiloxane (PDMS) film. (c) 30 min of inkabsorption time and 50 55 \% humidity were optimal conditions for inking. 

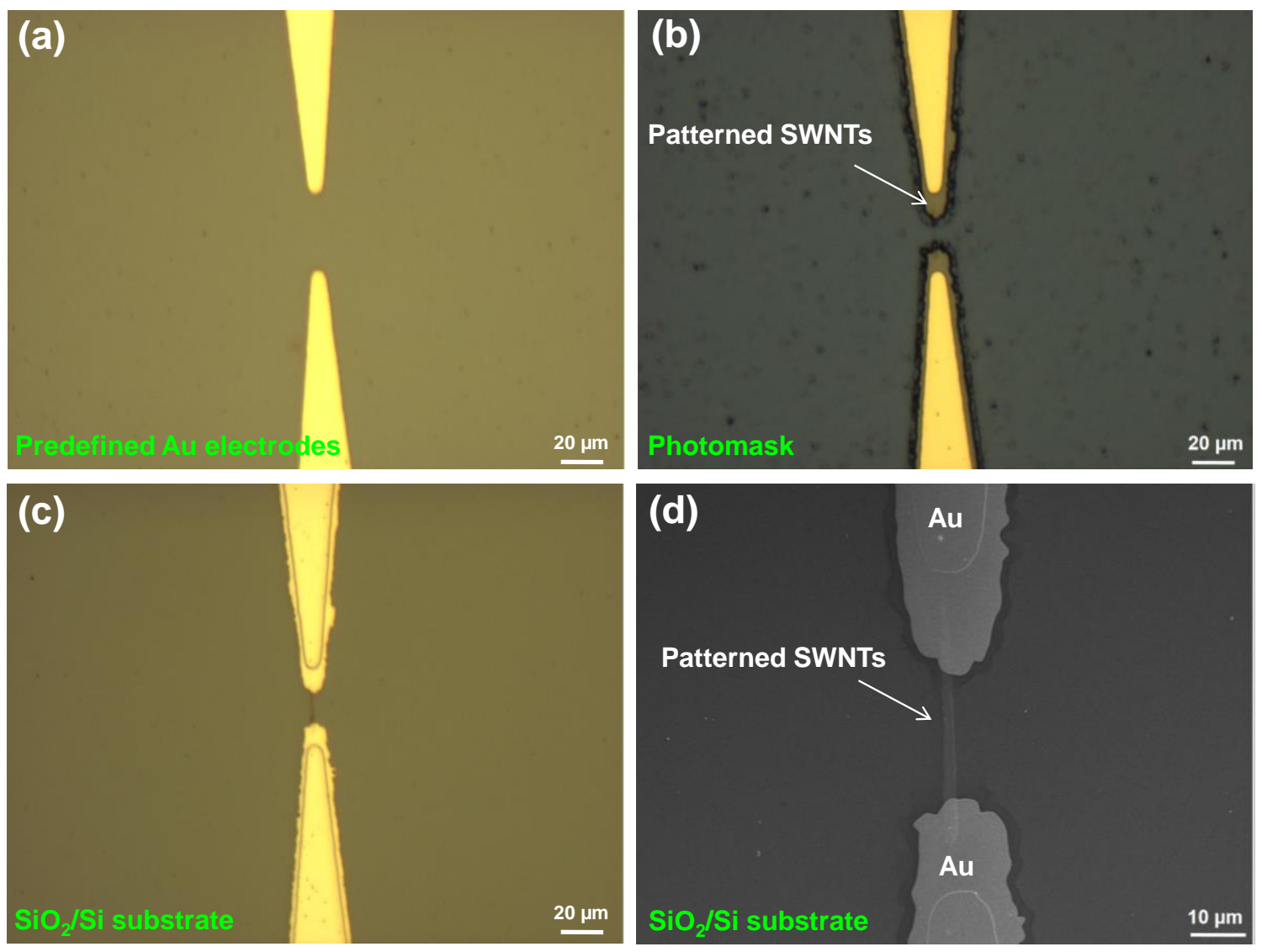

Figure S2. Stepwise evaluation of SWNTs based electrodes fabrication. (a) Optical image of a Au source and drain electrode. The Au electrodes were defined by photolithography using a conventional lift-off technique. (b) Optical image of the SWNTs patterned in between the Au source and drain electrode by the direct-write DPN method. After the DPN patterning, photoresist (AZ 7220) was deposited surrounding the pre-defined Au electrode for the additional Au deposition to make ohmic contact. (c and d) Optical and scanning electron microscopy (SEM) images of the final SWNTs patterned electrode. DPN patterned SWNTs remained intact after the thorough washing processes with acetone, methanol, and deionized water. 


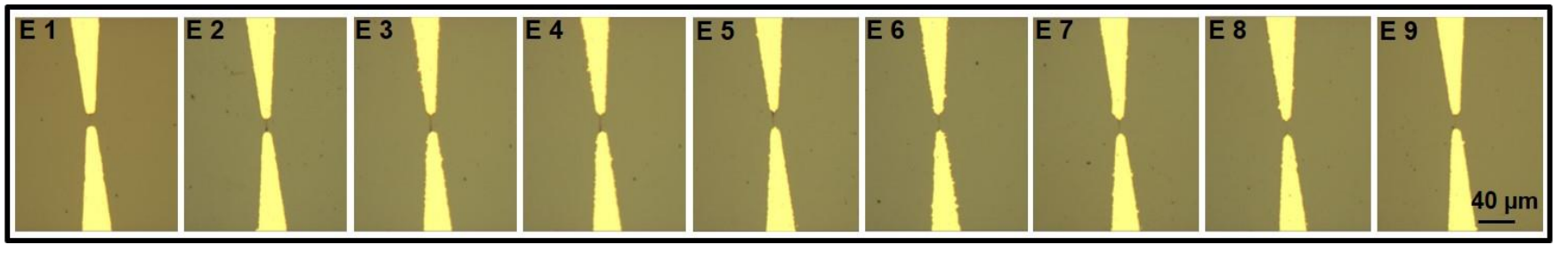

Figure S3. Multi-arrays of SWNTs were directly patterned onto several circuits across gap electrodes after single inking of SWNT/DPN ink. 